\title{
Évaluation de l'activité antimicrobienne de différents extraits d'Helichrysum stoechas subsp. rupestre
}

Manuscrit reçu le 3 janvier 2016 et accepté le 18 août 2016

\section{Evaluation of antimicrobial activity of different extracts of Helichrysum stoechas subsp. rupestre}

\author{
Farah HADDOUCHI ${ }^{1}$, Khadidja ZERHOUNI ${ }^{2}$, Adel SIDI-YEKHELEF ${ }^{3}$ et
}

Tarik Mohammed CHAOUCHE ${ }^{1 *}$

${ }^{1}$ Laboratoire de produits naturels, Département de Biologie, Faculté des Sciences de la Nature et de da Vie et Sciences de la Terre et de l'Univers, Université Abou Bekr Belkaïd, Tlemcen, 13000, Algérie.

${ }^{2}$ Laboratoire de microbiologie moléculaire et protéomique et santé, Département de Biologie, Faculté des Sciences de la Nature et de la Vie, Université Djillali Liabès, Sidi Bel Abbes, 22000, Algérie.

3 Laboratoire de la population et développement durable en Algérie, Département de démographie, Faculté des Sciences Humaines et Sociales, Université Abou Bekr Belkaïd, Tlemcen, 13000, Algérie.

\section{Résumé}

Les plantes médicinales constituent une source riche et diversifiée de métabolites secondaires, qui ont une application commerciale dans les domaines pharmaceutiques et biomédicaux. Notre travail s'inscrit dans le cadre de la recherche de nouveaux antimicrobiens naturels à partir d'une plante de la région de Tlemcen. Il s'agit des fleurs et des tiges feuillées d'Helichrysum stoechas subsp. rupestre. Les extractions successives, par des solvants de polarités croissantes (hexane, dichlorométhane, méthanol et aqueux), ont abouti à des rendements en extraits méthanoliques et aqueux beaucoup plus importants par rapport aux solvants de faible polarité. Les résultats de l'étude de l'activité antimicrobienne, réalisée par la méthode des disques et celle des dilutions sur milieu liquide, montrent que quelques extraits, à l'exception des extraits aqueux, sont doués d'une activité antibactérienne modérée. Les souches les plus sensibles sont Bacillus cereus, Acinetobacter baumanii, Proteus mirabilis, Staphylococcus aureus, Enterococcus faecalis et Escherichia coli, avec des diamètres compris entre 11 et $13 \mathrm{~mm}$. Les valeurs de la concentration minimale inhibitrice (CMI) ont montré un large éventail de valeurs (jusqu'à $25 \mathrm{mg} / \mathrm{ml}$ ) en comparaison avec les zones d'inhibition (13 $\mathrm{mm})$. Les extraits des deux plantes n'ont montré aucune activité antifongique.

Mots clés: Helichrysum stoecha subsp. Rupestre, polarité, activité antimicrobienne, CMI

\footnotetext{
*Adresse électronique pour la correspondance : tarik.chaouche@mail.univ-tlemcen.dz
} 


\begin{abstract}
Medicinal plants are a rich and diverse source of secondary metabolites, which have a commercial application in the pharmaceutical and biomedical industry. Our work is part of the search for new natural antimicrobial from parts of a plant in the region of Tlemcen. These are flowers and leafy stems of Helichrysum stoechas subsp.rupestre. Results of the successive extractions with solvents of increasing polarity (hexane, dichloromethane, methanol and aqueous), show that the yields of methanolic and aqueous extracts are much larger compared to those of low polarity solvents. The results of the study of the antimicrobial activity, carried out by the disc method and the dilutions in liquid medium show that some extracts, with the exception of aqueous extracts, are endowed with a moderate antibacterial activity. The most sensitive strains are Bacillus cereus, Acinetobacter baumanii, Proteus mirabilis, Staphylococcus aureus, Enterococcus faecalis, and Escherichia coli, with diameters of 11 and $13 \mathrm{~mm}$. Minimal inhibitory concentration (MIC) values show a wide range of values (up to 25 $\mathrm{mg} / \mathrm{ml}$ ) compared with the zones of inhibition $(13 \mathrm{~mm})$. The extracts of the two plants showed no antifungal activity.
\end{abstract}

Keywords: Helichrysum stoecha subsp. Rupestre, polarity, antimicrobial activity, MIC.

\title{
1. Introduction
}

Les plantes médicinales ont toujours eu une place importante dans l'arsenal thérapeutique de l'humanité. Elles constituent une source importante de molécules bioactives qui font généralement partie des métabolites secondaires [1]. Face à l'apparition de formes résistantes de plusieurs bactéries à certains antibiotiques, la recherche de nouvelles molécules actives et à large spectre d'action est devenue une nécessité [2]. Une des stratégies pour cette recherche consiste à explorer les plantes utilisées en médecine traditionnelle [3].

Le choix est porté sur une plante médicinale Helichrysum stoechas subsp. rupestre caractérisée par des capitules en corymbe peu nombreux. Feuilles tomenteuses en-dessus (bien que vertes), très blanches en-dessous. Bractées de l'involucre aiguës, les intérieurs linéaires à partie coriace couverte de glandes dorées [4].

Cette plante contient des terpènes, des huiles essentielles, des flavonoïdes [5], des acides phénoliques, des coumarines et des micronutriments $(\mathrm{Cu}, \mathrm{Mn}, \mathrm{Zn})$ [6-7].

L'objectif de cette étude est d'évaluer l'activité antimicrobienne des fleurs et des tiges feuillées d' Helichrysum stoechas subsp. rupestre extrait avec plusieurs solvants de polarité croissante et la détermination de la concentration minimale inhibitrice des souches qui ont montré une certaines sensibilité. 


\section{Matériel et méthodes}

\subsection{Matériel végétal}

Helichrysum stoechas subsp. rupestre a été récoltée en mois de mars 2013 (période de floraison). Dans la région de Honaïne à environ $44 \mathrm{~km}$ au Nord-Ouest de Tlemcen (Algérie). Après séchage à l'abri des rayons solaires, afin de préserver au maximum l'intégrité des molécules, les fleurs et les tiges feuillées de cette espèce ont été séparées et broyées grossièrement dans un moulin électrique.

\subsection{Extraction par des solvants organiques à polarité croissante}

L'extraction est effectuée par épuisements successifs du matériel végétal par un extracteur Soxhlet sous agitation continue durant 24 heures, en utilisant quatre solvants à polarité croissante : hexane (Hx), dichlorométhane (D), méthanol (M) et eau (A). La méthode a été décrite par Chaouche et al. [8]. Cette technique est retenue car elle favorise une extraction relativement complète des métabolites présents dans la matrice végétale [9].

Le volume de solvant doit être approprié à la quantité de matière végétale. Un rapport de 1/4 à 1/6 (P/V) est adopté selon la capacité d'absorption du solvant par la matière végétale. Après le passage de chaque solvant, les extraits sont évaporés à l'aide d'un évaporateur rotatif. L'extrait aqueux était, pour sa part, lyophilisé grâce à un lyophilisateur.

Nous pouvons déterminer le rendement en extrait sec en calculant la perte de poids en pourcentage de la matière sèche de départ :

$$
\operatorname{Rdt}(\%)=[\mathrm{P} 1-\mathrm{P} 2 / \mathrm{P} 3] \times 100
$$

où :

$\mathrm{P} 1$ : Poids du ballon après évaporation ;

P2 : Poids du ballon avant évaporation (ballon vide) ;

P3 : Poids de la matière végétale sèche de départ.

Les rendements ont été énoncés dans la moyenne \pm écart type de trois répétitions. L'extrait sec est pesé et repris dans quelques millilitres de DMSO pour l'évaluation de l'activité antimicrobienne. Les échantillons ont été conservés à $4{ }^{\circ} \mathrm{C}$ pour une utilisation ultérieure [10].

\subsection{Détermination de l'activité antimicrobienne}

L'activité antimicrobienne des extraits a été évaluée en utilisant des souches de référence de laboratoire (American Type Culture Collection "ATCC" pour les bactéries et Candida albicans, Musé National d'Histoire Naturelle "MNHN" pour les champignons filamenteux), obtenues par le Laboratoire des produits naturels, département de Biologie (Université de Tlemcen, Algérie) :

- Bactéries à Gram positif: Bacillus cereus (ATCC 10876), Enterobacter cloacea (ATCC 13047), Enterococcus faecalis (ATCC 49452), Listeria monocytogenes (ATCC15313), Staphylococcus aureus (ATCC 25923).

- Bactéries à Gram négatif: Acinetobacter baumanii (ATCC 19606), Escherichia coli (ATCC 25922), Klebsiella pneumoniae (ATCC 700603), Pseudomonas aeruginosa 
(ATCC 27853), Proteus mirabilis (ATCC 35659), Salmonella typhimurium (ATCC 13311), Citrobacter freundii (ATCC 8090).

- Champignon non filamenteux : Candida albicans (ATCC 444), Candida albicans (ATCC 10231).

- Champignons filamenteux : Aspergillus fumigatus (MNHN 566), Aspergillus flavus (MNHN 994294), Fusarium oxysporum (MNHN 963917).

Les souches ont été revivifiées et la turbidité a été ajusté à $0,5 \mathrm{McF}$ arland, ce qui correspond à $1-2 \times 10^{8} \mathrm{UFC} / \mathrm{ml}$ pour les bactéries $(\mathrm{D} . \mathrm{O}=0,08$ à $0,1 / \lambda=625 \mathrm{~nm}), 1-5 \times 10^{6} \mathrm{UFC} / \mathrm{ml}$ pour C. albicans $\left(\mathrm{D} . \mathrm{O}=0,12\right.$ à $0,15 / \lambda=530 \mathrm{~nm}$ ) [11] et $10^{6}$ spores $/ \mathrm{ml}$ pour les champignons filamenteux (68-82\% de transmittance $/ \lambda=530 \mathrm{~nm})$ [12]. Les cultures ont été diluées avec du bouillon Mueller-Hinton pour les bactéries, du bouillon Sabouraud pour C. albicans et une solution saline stérile pour les souches fongiques, afin d'atteindre des densités optiques correspondantes pour chaque test.

Deux méthodes différentes ont été utilisées pour la détermination de l'activité antimicrobienne, in vitro : une méthode de diffusion en disque dans un milieu gélosé et les méthodes de dilution (méthode de micro-dilution en bouillon pour les bactéries et $C$. albicans, la méthode de dilution en milieu gélosé pour les champignons), selon le Comité national des normes du laboratoire clinique [11]. Les zones d'inhibition et les concentrations minimales inhibitrices (CMI) de la gentamicine et de l'amphotéricine B ont été également déterminées dans des expériences parallèles afin de contrôler la sensibilité des micro-organismes d'essai. Tous les tests ont été effectués en triple.

\subsubsection{Méthode de diffusion en disque}

Les extraits ont été testés pour leur activité antimicrobienne par la méthode de diffusion en disques, en utilisant $100 \mu \mathrm{l}$ de suspension des microorganismes testés, contenant $2 \times 10^{8} \mathrm{UFC}$ / $\mathrm{ml}$ pour les bactéries, $1-5 \times 10^{6} \mathrm{UFC} / \mathrm{ml}$ pour C. albicans et $2 \times 10^{5}$ spores / $\mathrm{ml}$ pour les souches fongiques. Les milieux Mueller-Hinton et Sabouraud gélosés, stériles et refroidis jusqu'à $45-50^{\circ} \mathrm{C}$, ont été distribués dans des boîtes de Pétri stériles de $9 \mathrm{~cm}$ de diamètre (15 $\mathrm{ml})$. Les disques de papier filtre (6 $\mathrm{mm}$ de diamètre) ont été individuellement imprégnés avec $5 \mu \mathrm{l}$ de l'extrait $(500 \mu \mathrm{g} /$ disque) et ensuite placés sur la surface des milieux gélosés déjà inoculés avec les microorganismes testés.

Les boîtes de Pétri ont été conservés à $4{ }^{\circ} \mathrm{C}$ pendant $2 \mathrm{~h}$ et ont été ensuite incubées à $37^{\circ} \mathrm{C}$ pendant $24 \mathrm{~h}$ pour les bactéries, à $30{ }^{\circ} \mathrm{C}$ pendant $24 \mathrm{~h}$ pour $C$. albicans et $48 \mathrm{~h}$ pour les souches de champignons. Les diamètres des zones d'inhibition $(\mathrm{mm})$ ont été mesurés, y compris le diamètre des disques [13]. La gentamicine (15 $\mu \mathrm{g} /$ disque) et l'amphotéricine $\mathrm{B}$ (20 $\mu \mathrm{g} /$ disque) ont servi de témoins positifs.

\subsubsection{Détermination de la concentration minimale inhibitrice (CMI)}

Pour les bactéries et $C$. albicans, la méthode de micro-dilution en bouillon a été utilisée pour déterminer la CMI. Tous les tests ont été effectués dans le milieu Mueller Hinton en bouillon. Les extraits étudiés ont été dissous dans le diméthylsulfoxyde (DMSO) à $1 \%$, puis dilués à la 
concentration la plus élevée. Des dilutions en série ont été préparées dans une microplaque de microtitration de 96 puits dans la gamme de concentrations choisie. Les souches, dont la concentration finale a été ajusté à $5 \times 10^{5} \mathrm{UFC} / \mathrm{ml}$ pour les bactéries et à $2,5 \times 10^{6} \mathrm{UFC} / \mathrm{ml}$ pour $C$. albicans, sont ajoutées dans chaque puits. Les bactéries et $C$. albicans ont été respectivement incubées, à $37^{\circ} \mathrm{C}$ et à $30^{\circ} \mathrm{C}$, pendant $24 \mathrm{~h}$. La CMI est définie comme la plus faible concentration de l'extrait à laquelle le micro-organisme ne montre pas une croissance visible. La croissance des microorganismes a été indiquée par la turbidité. La gentamicine a été utilisée comme composé de référence.

Pour les champignons filamenteux, les CMI ont été déterminées par la méthode de dilution en milieu gélosé [14]. Les souches testées ont été cultivées dans l'agar de dextrose de pomme de terre (PDA), dans des boîtes de Pétri, pendant 5-7 jours. Les extraits testés, dissous dans du DMSO à $1 \%$, ont été utilisé à différentes concentrations. Chaque concentration a été mélangée avec le milieu PDA semi-solide et stérile et ensuite versé dans des boîtes de Pétri stériles (15 $\mathrm{ml}$ dans chaque plaque). Un disque de $6 \mathrm{~mm}$ de diamètre de la gélose recouverte de mycélium a été placé sur la surface de la gélose. Les plaques ont été incubées pendant 5-7 jours à $28{ }^{\circ} \mathrm{C}$. Deux répétitions ont été faites pour chaque test. L'amphotéricine B a été utilisée comme composé de référence.

\section{Résultats}

\subsection{Rendements des extractions}

Les extractions successives par des solvants de polarité croissante permettent de séparer les composés de la matière végétale selon leur degré de solubilité dans le solvant d'extraction.

Les différents rendements obtenus, ainsi que les aspects et les couleurs des différents extraits, sont présentés dans le tableau 1.

Tableau 1. Aspects, couleurs et rendements massique (\%) des extraits obtenus par extraction au Soxhlet

\begin{tabular}{ccccc}
\hline $\begin{array}{c}\text { Parties de la } \\
\text { plante }\end{array}$ & Solvants & Aspect & Couleur & $\begin{array}{c}\text { Rendement } \\
\%\end{array}$ \\
\hline \hline \multirow{2}{*}{ Fleurs } & Hexane & Visqueux & Vert & jaunâtre \\
& Dichlorométhane & Visqueux & Vert clair & 0,765 \\
& Méthanol & Visqueux & Jaune & 24,01 \\
& Aqueux & Poudre & Marron & 9,65 \\
\hline \multirow{4}{*}{ Tiges feuillées } & Hexane & Visqueux & Vert & 2,44 \\
& Dichlorométhane & Poudre & Vert foncé & 1,17 \\
& Méthanol & Visqueux & Jaune & 8,017 \\
& Aqueux & Poudre & Marron & 11,97 \\
\hline
\end{tabular}

Les extraits méthanoliques et aqueux ont les rendements les plus élevés par rapport à ceux d'hexane et de dichlorométhane. Le rendement en extrait du méthanol est plus élevé dans le cas de la partie des fleures $d^{\prime} H$. stoechas subsp. rupestre. 


\section{2. Étude de l'activité antimicrobienne}

3.2.1. Méthode de diffusion des disques sur milieu gélosé

Les souches bactériennes se comportent différemment vis-à-vis de tous les extraits. Les souches de Citrobacter freundii, Enterobacter cloacea, Klebsiella pneumoniae et Pseudomonas aeruginosa, se sont montrées résistantes à tous les extraits.

Bacillus cereus et Acinetobacter baumanii vis-à-vis des extraits d'hexane des fleurs et des tiges feuillées et Proteus mirabilis vis-à-vis de l'extrait d'hexane des fleurs, avec des diamètres de $13 \mathrm{~mm}$.

Staphylococcus aureus et Escherichia coli vis-à-vis de l'extrait du méthanol des tiges feuillées, avec des diamètres de $12 \mathrm{~mm}$.

Enterococcus faecalis, Staphylococcus aureus et Escherichia coli vis-à-vis de l'extrait d'hexane des tiges feuillées d' H. stoechas subsp. rupestre, avec des diamètres de $11 \mathrm{~mm}$.

Les résultats de cette activité, montrent qu'aucun des extraits n'a une activité antifongique sur les champignons filamenteux et sur les deux souches de Candida albicans, testés.

\subsubsection{Détermination des Concentrations Minimale Inhibitrice}

Pour les souches qui ont montré une certaines sensibilité $(11-13 \mathrm{~mm})$, nous avons déterminé les CMI. Les résultats sont représentés dans le tableau, pour les extraits $(\mathrm{mg} / \mathrm{ml})$ et l'antibiotique de référence, Gentamicine $(\mu \mathrm{g} / \mathrm{ml})$.

Tableau 2. Concentrations Minimales Inhibitrices.

\begin{tabular}{ccccc}
\hline & $\begin{array}{c}\text { HSF } \\
\text { Hexane }\end{array}$ & $\begin{array}{c}\text { HSTF } \\
\text { Hexane }\end{array}$ & $\begin{array}{c}\text { HSTF } \\
\text { Méthanol }\end{array}$ & Gentamicine \\
\hline \hline Staphylococcus & $/$ & $3,12-6,25$ & $/$ & $0,25-0,5$ \\
aureus & $/$ & $0,78-1,56$ & $3,12-6,25$ & $4-8$ \\
$\begin{array}{c}\text { Enterococcus faecalis } \\
\text { Proteus mirabilis }\end{array}$ & $1,56-3,12$ & $/$ & $/$ & $1-2$ \\
Escherichia coli & $/$ & 25 & $/$ & $0,5-1$ \\
$\begin{array}{c}\text { Bacillus cereus } \\
\text { Acinetobacter }\end{array}$ & $1,56-3,12$ & $12,5-25$ & $/$ & $0,25-0,5$ \\
baumanii & 25 & $0,78-1,56$ & $/$ & $>8$ \\
\hline
\end{tabular}

HSF : fleurs d' H. stoechas subsp. rupestre, HSTF : tiges feuillees d' H. stoechas subsp. rupestre, PSTF : tiges feuillées $P$. saxatile subsp. saxatile, / : non testés

Les valeurs des concentrations minimales inhibitrices ont été déterminées dans une large gamme de concentration allant de 0,78 à $25 \mathrm{mg} / \mathrm{ml}$. L'extrait le plus actif, avec les concentrations les plus faibles $(0,78-1,56 \mathrm{mg} / \mathrm{ml})$, est celui de l'hexane des tiges feuillées 
contre Enterococcus faecalis et Acinetobacter baumanii suivi par l'extrait de l'hexane de fleurs contre Proteus mirabilis et Bacillus cereus $(1,56-3,12 \mathrm{mg} / \mathrm{ml})$.

\section{Discussion}

Des études ont été consacrées à la détermination de ce pouvoir de certains extraits du genre Helichrysum, originaires de l'Afrique du sud. Celle réalisée par Meyer et Dilika. [15], sur plusieurs extraits d' $H$. pedunculatum, préparés séparément, montre que l'extrait de dichlorométhane a été le plus actif suivi par l'extrait aqueux. Celui préparé à partir du méthanol était totalement inactif. D'autres études réalisées sur cinq espèces d' Helichrysum, montrent que les extraits d'acétone et du méthanol d' $H$. dasyanthum, H. felinum, H. excisum et $H$. petiolare [16] et d'hexane d'H. tenax [17] sont actifs contre Bacillus cereus, les quatre premières espèces contre Staphylococcus aureus et la cinquième sur Staphylococcus epidermidis.

Donc, il n'existe pas une relation directe entre l'activité antimicrobienne et la polarité des solvants (dichlorométhane, eau, méthanol, acétone, hexane) utilisés pour nos extractions [18] et de plusieurs espèces d'Helichrysum étudiés par Meyer et Dilika [15] et Lourens et al. [16].

Les valeurs de CMI ont montré un large éventail de valeurs (jusqu'à $25 \mathrm{mg} / \mathrm{ml}$ ) en comparaison avec les zones d'inhibition $(13 \mathrm{~mm})$. Ceux-ci suggèrent que la taille de la zone d'inhibition ne reflète pas la réelle efficacité antibactérienne d'un composé. Ce point était en accord avec les suggestions de Cimanga et al. [19]. Mais selon Tegos et al. [20], ces valeurs de CMI ne signifient pas que tous les extraits testés soient inactifs.

\section{Conclusion}

Cette recherche a permis de mettre en évidence l'effet antimicrobien des extraits organiques des fleurs et des tiges feuillées d'Helichrysum stoechas subsp. rupestre. Les extraits méthanolique et héxanique ont montré une activité antibactérienne modérée. Toutefois, d'autres études seront nécessaires pour l'isolement et la caractérisation des composés responsables de cette activité et l'identification de leur mécanisme d'action in vivo.

\section{Références}

1. Haddouchi F, Chaouche TM, Ksouri R, et al. (2014) Phytochemical screening and in vitro antioxidant activities of aqueous-extracts of Helichrysum stoechas subsp. rupestre and Phagnalon saxatile subsp. saxatile. Chin J Nat Med 12(6): 415-22

2. Haddouchi F, Chaouche TM, Zaouali Y, et al. (2013) Chemical composition and antimicrobial activity of the essential oils from four Ruta species growing in Algeria. Food Chem 141(1): 253-8

3. Chaouche TM, Haddouchi F, Ksouri R, et al. (2013) In vitro evaluation of antioxidant activity of the hydro-methanolic extracts of Juniperus oxycedrus subsp. oxycedrus. Phytothérapie 11(4):244-9 
4. Quezel P, Santa S (1963) Nouvelle flore de l'Algérie et des régions désertiques méridionales. Edition Centre national de la recherche scientifiques, Tome II

5. Czinner E, Hagymasi K, Blazovics AK, et al. (2000) In vitro Antioxidant properties of Helichrysum arenarium (L.) Moench. J Ethnopharmacol 73(3): 437-43

6. Dombrowicz E, Swiatek L, Kopycki W. (1994) Phenolic acids in Inflorescentia Helicrysum and herba Hieracii pilosellae. Pharmazie 47: 469-70

7. Hwisa N, Auzi A, Parvez N, et al. (2011) Antinociceptive effect of Helichrysum stoechas in experimental animals. IJPI's J Pharmacol Toxicol 1(2): 23-7

8. Chaouche TM, Haddouchi F, Atik-Bekara F et al. (2015) Antioxidant, haemolytic activities and HPLC-DAD-ESI-MS ${ }^{\mathrm{n}}$ characterization of phenolic compounds from root bark of Juniperus oxycedrus subsp. Oxycedrus. Ind Crop Prod 64: 182-7

9. Romanik G, Gilgenast E, Przyjazny A, et al. (2007) Techniques of preparing plant material for chromatographic separation and analysis. J Biochem Biophys Methods 70(2): 25361

10. Mezouar D, Lahfa FB, Abdelouahid DE, et al. (2014) Activité antimicrobienne d'extraits d'écorce de racines de Berberis vulgaris. Phytothérapie 12: 380-5

11. National Committee for Clinical Laboratory Standards (NCCLS) (2001) Performance standards for antimicrobial susceptibility testing: eleventh informational supplement, M100- S11, Wayne, PA, USA

12. Pfaller MA, Messer SA, Karlsson $\AA$ et al. (1998) Evaluation of the Etestmethod for determining fluconazole susceptibilities of 402 clinical yeast isolates by usingthree different agar media. J Clin Microbiol 36(9): 2586-9

13. Biyiti LF, Meko'o DJL, Tamzc v et al. (2004) Recherche de l'Activité Antibactérienne de Quatre Plantes Médicinales Camerounaises. Trad pharmacol med Afr 13: 11-20.

14. Merghache D, Boucherit-Atmani Z, Boucherit K (2012) Évaluation de l'activité antifongique de différents extraits de la cannelle de Chine (Cinnamomum cassia). Phytothérapie 10: 215-21

15. Meyer JJM, Dilika F (1996) Antibacterial activity of Helichrysum pedunculatum used in circumcision rites. J Ethnopharmacol 53(1): 51-4

16. Lourens ACU, Reddy D, Baser KHC et al. (2004) In vitro biological activity and essential oil composition of four indigenous South African Helichrysum species. J Ethnopharmacol 95(2-3): 253-8

17. Drewes SE, Van Vuuren SF (2008) Antimicrobial acylphloroglucinols and dibenzyloxy flavonoids from flowers of Helichrysum gymnocomum. Phytochem 69: 1745-9

18. Ali-Shtayeh MS, Abu-Ghdeib SI (1999) Antifungal activity of plant extracts against dermatophytes. Mycoses 42(11-12): 665-72

19. Cimanga K, Kambu K, Tona L, et al. (2002) Correlation between chemical composition and antibacterial activity of essential oils of some aromatic medicinal plants growing in the Democratic Republic of Congo. J Ethnopharmacol 79(2): 213-20

20. Tegos G, Stermitz FR, Lomovskaya O, et al. (2002) Multidrug pump inhibitors uncover remarkable activity of plant antimicrobials. Antimicrob Agents Chemother 46 (10): $3133-41$ 